\title{
Deficiency of cyclase-associated protein 2 promotes arrhythmias associated with connexin43 maldistribution and fibrosis
}

Florian Stöckigt ${ }^{1}$, Vivek Shahaji Peche ${ }^{2,3}$, Markus Linhart ${ }^{1}$, Georg Nickenig ${ }^{1}$, Angelika Anna Noegel2,3, Jan Wilko Schrickel ${ }^{1}$

\begin{abstract}
${ }^{1}$ Department of Medicine - Cardiology, University Hospital Bonn, Bonn, Germany ${ }^{2}$ Institute of Biochemistry I, University of Cologne, Cologne, Germany ${ }^{3}$ Center for Molecular Medicine Cologne (CMMC), Cologne Excellence Cluster on Cellular Stress Responses in Aging-Associated Diseases (CECAD), University of Cologne, Cologne, Germany
\end{abstract}

Submitted: 29 August 2014

Accepted: 9 November 2014

Arch Med Sci 2016; 12, 1: 188-198

DOI: 10.5114 /aoms.2015.54146

Copyright $\odot 2016$ Termedia \& Banach

\begin{abstract}
Introduction: Cyclase-associated protein 2 (CAP2) plays a major role in regulating the actin cytoskeleton. Since inactivation of CAP2 in a mouse model by a gene trap approach (Cap $\left.2^{\text {gt/gt }}\right)$ results in cardiomyopathy and increased mortality, we hypothesized that CAP2 has a major impact on arrhythmias and electrophysiological parameters.

Material and methods: We performed long-term-ECG recordings in transgenic CAP2 deficient mice (C57BL/6) to detect spontaneous arrhythmias. In vivo electrophysiological studies by right heart catheterization and ex vivo epicardial mapping were used to analyze electrophysiological parameters, the inducibility of arrhythmias, and conduction velocities. Expression and distribution of cardiac connexins and the amount of cardiac fibrosis were evaluated.

Results: Spontaneous ventricular arrhythmias could be detected in Cap $2^{\text {gt/gt }}$ during the long-term-ECG recording. Cap $2^{g t / g t}$ showed marked conduction delays at atrial and ventricular levels, including a reduced heart rate $(421.0$ $\pm 40.6 \mathrm{bpm}$ vs. $450.8 \pm 27.9 \mathrm{bpm} ; p<0.01)$, and prolongations of $\mathrm{PQ}(46.3$ $\pm 4.1 \mathrm{~ms}$ vs. $38.6 \pm 6.5 \mathrm{~ms} ; p<0.01)$, QRS (16.2 $\pm 2.6 \mathrm{~ms}$ vs. $12.6 \pm 1.4 \mathrm{~ms}$; $p<0.01)$, and QTc interval ( $55.8 \pm 6.0 \mathrm{~ms}$ vs. $45.2 \pm 3.3 \mathrm{~ms} ; p=0.02)$ in comparison to wild type mice. The $\mathrm{PQ}$ prolongation was due to an infra-Hisian conduction delay (HV: $9.7 \pm 2.1 \mathrm{~ms}$ vs. $6.5 \pm 3.1 \mathrm{~ms} ; p=0.02$ ). The inducibility of ventricular tachycardias during the electrophysiological studies was significantly elevated in the mutant mice (inducible animals: $88 \%$ vs. $33 \%$; $p=0.04)$. Cap $2^{g t / g t}$ showed more abnormal distribution of connexin $43 \mathrm{com}$ pared to WT $(23.0 \pm 4.7 \%$ vs. $2.9 \pm 0.8 \% ; p<0.01)$. Myocardial fibrosis was elevated in Cap $2^{\text {gt/gt }}$ hearts ( $9.1 \pm 6.7 \%$ vs. $5.5 \pm 3.3 \%$; $p<0.01$ ).

Conclusions: Loss of CAP2 results in marked electrophysiological disturbances including impaired sinus node function, conduction delays, and susceptibility to malignant arrhythmias. Structural changes in Cap $2^{\text {gt/gt }}$ are associated with alterations in myocardial connexins and fibrosis.
\end{abstract}

Key words: mouse model, cardiomyopathy, arrhythmia, electrophysiology.

\section{Introduction}

The regulation and remodeling of the actin cytoskeleton depends on actin binding proteins that organize the actin filaments into specific struc-

\section{Corresponding author:} Dr. Florian Stöckigt Universitätsklinik Bonn Medizinische Klinik II Kardiologie

Sigmund Freud Str. 25

53105 Bonn, Germany

Phone: +49228 28715507

Fax: +4922828712329

E-mail: florian.stoeckigt@

ukb.uni-bonn.de 
tures [1]. One of these proteins is the cyclase-associated protein (CAP), which belongs to the family of G-actin sequestering proteins [2]. It is essential for maintaining the balance between G- and F-actin. The CAPs are highly conserved proteins that have been described in yeast, plants, Dictyostelium, Drosophila and mammals. The CAP deficiency results in defects in vesicle trafficking, endocytosis, and in an altered cell morphology and cell growth [3].

Two closely related homologs of CAP have been described in mammals: CAP1 is expressed in nearly all cells, and at a subcellular level it is present in regions with high actin dynamics [4]. CAP2 expression is restricted to a limited number of tissues and is mainly found in brain, skin, skeletal and cardiac muscle. The occurrence of CAP2 in muscle cells varies during development: during myogenesis CAP2 is predominantly a nuclear protein, whereas in differentiated muscles it is localized at the $\mathrm{M}$-line of the sarcomere [5].

$A$ recent study demonstrated that CAP2 is an essential component of the cardiac sarcomere. Deletion of CAP2 in a mouse model resulted in a reduced number of myocytes, shortening of the sarcomere and disarrangement in sarcomere structure with indistinguishable $M$-lines and I-bands. Mice lacking CAP2 exhibited a decreased survival rate and dilated cardiomyopathy without hypertrophy associated with bradycardia and atrioventricular conduction delays [6].

Mutations in sarcomeric proteins such as actin, troponin, myosin, and desmin play an important role in cardiomyopathies [7]. Alterations of the cardiac cytoskeleton have also been shown to have an impact on cardiac electrophysiology [8], and mutations in CAP2 might therefore be a so far unrecognized cause of human cardiomyopathy.

In this study, we aim to investigate the incidence and susceptibility to arrhythmias and associated structural abnormalities due to this mutation in a mouse model with a genetically induced inactivation of CAP2.

\section{Material and methods}

\section{Transgenic animals}

The studies were performed in transgenic mice (C57BL/6; age $16.5 \pm 2.5$ weeks) with a heterozygous (Cap $2^{g t /+}$ ) and homozygous (Cap $2^{\text {gt/gt }}$ ) Cap2 inactivation. The generation of CAP2 knockout mice using a gene trap approach has been described previously [6]. All procedures were performed in accordance with institutional guidelines and the German animal protection law, and conformed to the Guide for the Care and Use of Laboratory Animals published by the US National Institutes of Health (NIH Publication No. 85-23, revised 1996).

\section{Surface ECG and electrophysiological investigation}

In vivo transvenous electrophysiological (EP) investigations were carried out using a 2-French octapolar mouse EP catheter (Ciber Mouse, NuMed Inc., NY, USA) as described before [9]. In brief, anesthesia was performed with inhaled isoflurane (1.2 vol.\% isoflurane in $\left.70 \% \mathrm{~N}_{2} \mathrm{O} / 30 \% \mathrm{O}_{2}\right)$. After dissecting the jugular vein, the catheter was positioned in the right cardiac cavities. The intracardiac ECG was analyzed for the AH (from first atrial signal to His signal) and HV (from His to first ventricular signal) interval.

Electrophysiological investigations included the determination of functional EP parameters using a modified multi-programmable stimulator (Model 5328; Medtronic, MN, USA). Sinus node recovery time (SNRT) was defined as the maximum return cycle length after $10 \mathrm{~s}$ fixed-rate atrial pacing at S1S1 cycle length 120 ms. Wenckebach periodicity (WBP) and 2:1 AV nodal block (2: 1 block) were evaluated by fixed rate atrial pacing (10 s fixed rate at S1S1: $120 \mathrm{~ms}, 5 \mathrm{~ms}$ stepwise reduction). The WBP was defined as the longest S1S1 cycle length with loss of 1 : 1 AV nodal conduction; $2: 1$ block as the longest S1S1 with $2: 1$ AV nodal conduction.

Atrial, ventricular and AV nodal refractory periods (ARP, VRP and AVNRP) were evaluated by programmed stimulation maneuvers (7 stimuli fixed rate at S1S1 cycle length: 120 ms; one short coupled extrastimulus with a 5 ms stepwise S1S2 reduction). ARP was defined as the longest S1S2 with absent atrial response, AVNRP as the longest S1S2 with loss of AV nodal conduction, and determined after programmed atrial stimulation. The VRP was measured similar to ARP after ventricular extrastimulus pacing.

The inducibility of atrial fibrillation (AF) was tested by atrial burst stimulation ( $5 \mathrm{~S}$ at S1S1: 50-10 ms, $10 \mathrm{~ms}$ stepwise reduction; stimulus amplitudes 1.0 and $2.0 \mathrm{~mA}$; resulting in a total of 10 atrial burst stimulations per animal). The susceptibility to ventricular tachyarrhythmias (VTs) was determined by ventricular extrastimulus pacing (S1S1: $120 \mathrm{~ms}, 100 \mathrm{~ms}$, and $80 \mathrm{~ms}$ followed by up to 3 extra beats) and ventricular burst stimulation (1 s at S1S1: 50-10 ms, 10 ms stepwise reduction; stimulus amplitudes 1.0 and $2.0 \mathrm{~mA}$, resulting in a total of 19 ventricular burst stimulations per animal). The VTs were defined as $\geq 4$ consecutive ventricular ectopic beats.

\section{Long-term ECG recording}

Long-term ECG recordings were performed in 13 mice ( 5 WT, 4 Cap $2^{g t /+}$ and 4 Cap $2^{g t / g t}$ ) by implanted telemetry devices (Modell EA-F20; DataScienc- 
es International, St. Paul, MN). Under inhalation anesthesia as described above, the radiofrequency transmitter was inserted into a subcutaneous tissue pocket. The leads were fixed to the pectoral muscles in an Einthoven II position. Ten days after recovery from the surgical instrumentation the baseline recordings were performed in a constant environment. Thereafter a physical stress test (10 min swimming) was performed under continuation of ECG registration. LabChart 7pro (v7.3.3, AD Instruments) was used for a manually performed beat-to-beat analysis for the detection of arrhythmias. Signal averaged ECGs were calculated from 200 QRS complexes at a time for morphological analysis and calculations of electrocardiographic standard parameters.

\section{Langendorff perfusion and epicardial mapping}

Isolated hearts were Langendorff-perfused with Krebs-Henseleit buffer at constant pressure as previously described [9]. Epicardial activation mapping was performed with a 36-electrode array (EcoFlexMEA36, Multi-Channel-Systems, Reutlingen, Germany; electrode spacing: $300 \mu \mathrm{m}$ ). Conduction velocities in longitudinal and transversal myocardial fiber orientation were assessed after applied epicardial stimulation using Cardio-2D-Software (version 2.0.3, Multi-Channel-Systems, Reutlingen, Germany). A programmable stimulator (Stimulator 1, Hugo Sachs Elektronik, Germany) was used to apply square current pulses (duration $1 \mathrm{~ms}$, stimulus amplitude 2 times pacing threshold) at a cycle length of $120 \mathrm{~ms}$.

\section{RNA isolation and real-time PCR}

Myocardial samples were homogenized and incubated in Trizol. Precipitation of RNA was performed on ice. After measurement of the RNA (Nanodrop, PeqLab) the cDNA synthesis was performed using the Superscript III Vilo Kit (Invitrogen) according to the manufacturer's instructions. The semiquantitative real-time PCR for evaluation of connexin40 (Cx40) and Cx43 (assays by Invitrogen) was performed using the TaqMan Gene Expression Mastermix (Applied Biosystems). The threshold cycle values were calculated for each mRNA and differences between the groups were expressed as a percentage.

\section{Western blot}

Heart tissue was snap frozen in liquid nitrogen, ground in a mortar and homogenized in a lysis buffer. Samples were centrifuged and protein concentrations were determined by photometry. Supernatants were subjected to standard SDSPAGE and proteins were blotted to nitrocellulose membrane. The immunoblots were blocked with $3 \%$ BSA in PBST and incubated with appropriate primary antibodies (rabbit polyclonal antibodies against Cx43 (Sigma), mouse-monoclonal antibodies specific for Cx40 (Sigma), mouse monoclonal anti-Coronin 7 (K37-142-1) [10] and anti-Tubulin WA3 (kind gift from Dr. U. Euteneuer, München)) at $4^{\circ} \mathrm{C}$ overnight. Signal detection was performed with horseradish peroxidase-conjugated secondary antibodies, chemiluminescent processing $(\mathrm{ECL}$, Invitrogen), and autoradiography. Connexin band intensities were normalized to the relative intensity of the corresponding coronin 7/tubulin band for each sample. Densitometric analysis was done using ImageJ for quantification of relative protein levels of $\mathrm{Cx} 40$ and $\mathrm{Cx} 43$.

\section{Immunofluorescence}

Heart tissue was fixed in 4\% paraformaldehyde, embedded in paraffin and sectioned. After deparaffinization, antigen retrieval and blocking, sections were incubated with primary mouse-monoclonal antibodies specific for Cx43 (Sigma) followed by secondary antibodies (Invitrogen - Molecular Probes). F-actin was visualized with TRITC-phalloidin (Sigma). Sections were mounted and imaged with a Leica confocal microscope. Quantification of the signal intensity for $\mathrm{Cx} 43$ at the intercalated discs in relationship to maldis tributed and lateralized signals was performed by pixel analysis using a digital image analysis program (Adobe Photoshop V7.0). For this purpose we calculated the total number of immunofluorescent (Cx43-labeled) pixels in longitudinal myocyte sections at maximum magnification (400x) and determined the percentage of pixels that were not located within the intercalated disc area. Fifteen myocytes in 5 animals per group were studied, resulting in a total of 225 analyzed myocytes.

\section{Quantification of myocardial fibrosis}

Four-micrometer sections of cryo-conserved whole heart samples were stained with Sirius red $(0.1 \%$ in saturated aqueous picric acid; Sigma Aldrich). Four random fields of the left and right ventricle of each heart were analyzed for myocardial fibrosis in a four-chamber-view section of the heart. Cardiac fibrosis was defined as the percentage area of extracellular Sirius red staining and computed using a digital image analysis program (Adobe Photoshop, V7.0).

\section{Statistical analysis}

Data are expressed as mean \pm one standard deviation. All data were analyzed by one-way ANOVA; differences between all groups were assessed using Bonferroni's multiple comparison 
test. Discrete variables were analyzed by two-sided Fisher's exact test. Relationships were analyzed by two-tailed, non-parametric correlation analysis (Spearman). A $p$-value $<0.05$ was regarded as statistically significant.

\section{Results}

\section{Lack of CAP2 leads to spontaneous arrhythmias}

During the long-term-ECG recordings, which show cardiac rhythm uninfluenced by anesthetics, Cap2 $2^{\text {gt/gt }}$ displayed a reduced heart rate (WT: 598.5 \pm 35.9 beats per minute vs. Cap $2^{\text {gt/gt }}$ : $549.8 \pm 17.2$ beats per minute; $p=0.04$ ) and prolongations in $\mathrm{PQ}$ and QT interval compared to WT, as demonstrated in Figures $1 \mathrm{~A}-\mathrm{C}$. Cap $2^{\text {gt/ }}$ did not show significant changes in ECG parameters compared to WT.

The CAP2 ablation resulted in numerous spontaneous arrhythmias (Figures $1 \mathrm{E}-\mathrm{H}$ ): $\mathrm{Cap}^{\mathrm{gt} /+}$ showed spontaneous AV blocks, sinus arrests, and sinus arrhythmias during the baseline recording. Cap $2^{\text {gt/gt }}$ mice developed premature ventricular contractions and ventricular couplets which were not present in the WT controls. In all mice paroxysmal short time AV blocks could be detected; however, in WT mice only the absence of single QRS complexes was documented during periods of rest, whereas Cap $2^{\text {gt/gt }}$ presented with longer pauses up to $700 \mathrm{~ms}$ and AV blocks with up to 4 missing QRS complexes. Moreover, we detected paroxysmal idioventricular rhythms in $75 \%$ of Cap $2^{g t / g t}$ animals but never in WT. The stress test revealed polymorphic premature ventricular contractions and multiple AV blocks only in the mutant mice.

\section{Malignant ventricular arrhythmias can be induced in CAP2 deficient mice}

A significant reduction in basal heart rate and prolongations of $\mathrm{PQ}, \mathrm{QRS}$ and $\mathrm{QT}$ time were pres-

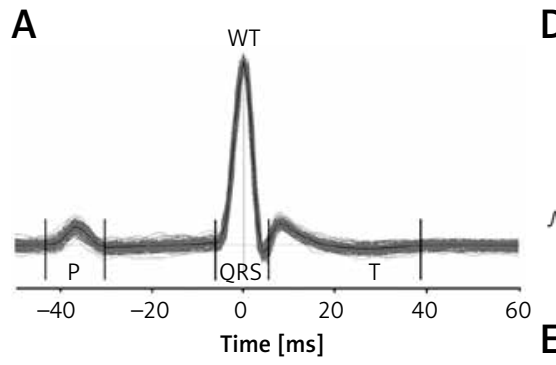

D WT
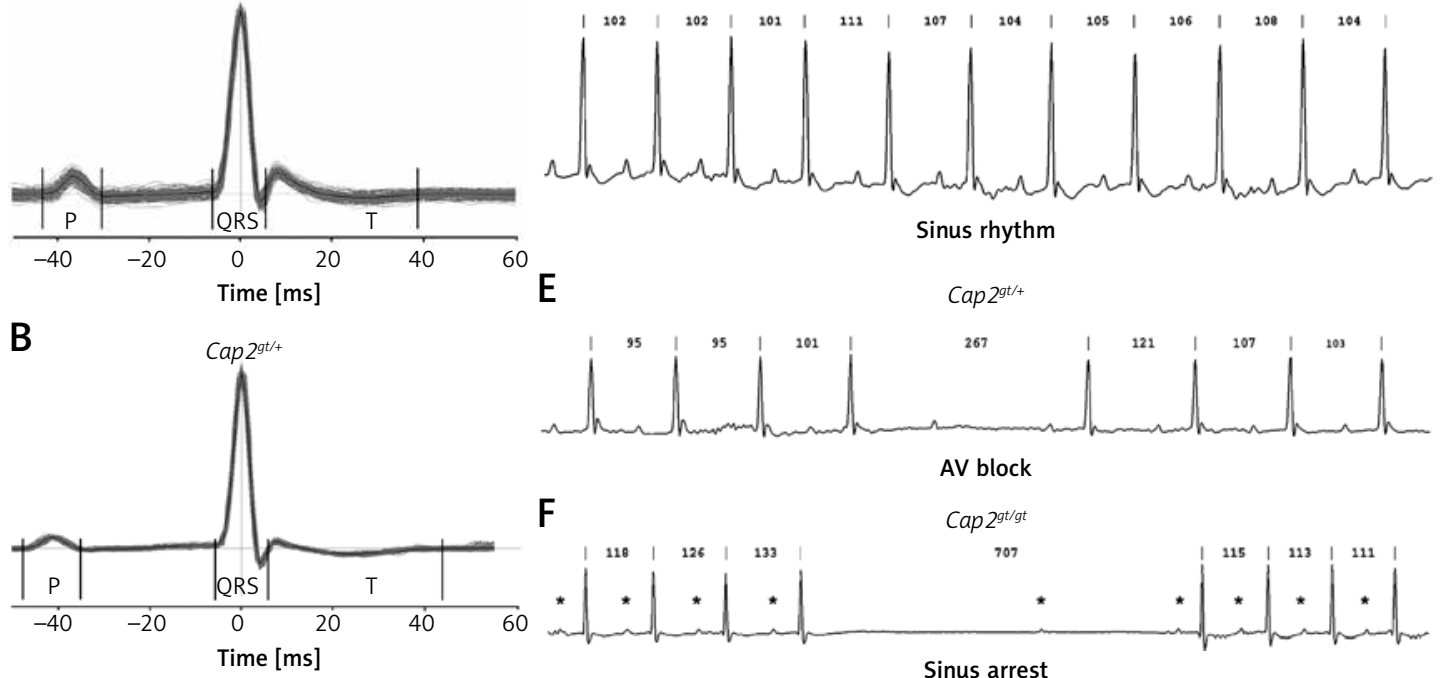

AV block
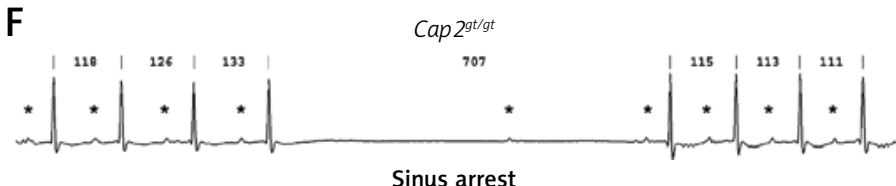

C

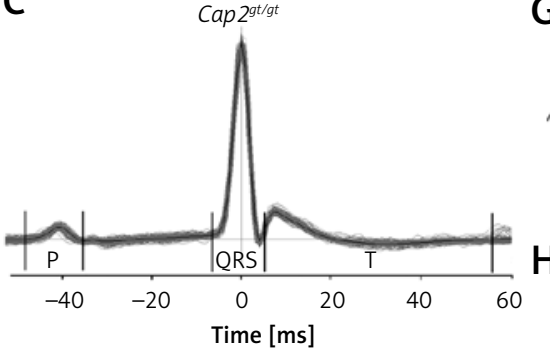

G

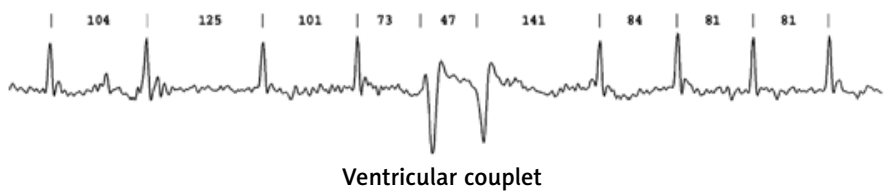

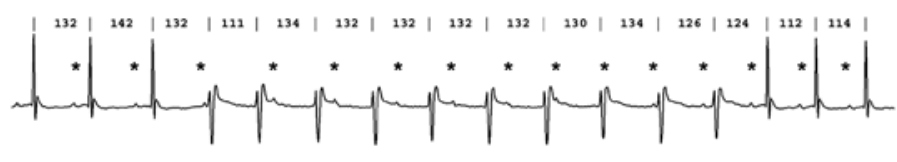

Junctional rhythm

Figure 1. Long-term ECG recordings. A-C - Signal averaged ECGs of A: WT, B: Cap 2 ${ }^{g t / 4}$, and C: Cap $2^{g t / g t}$ demonstrate successive prolongation in electrical conduction times in the mutant mice. D-H - Representative long-term-ECG recordings and sample traces of heart rhythm disorders. D - WT mice showed sinus rhythm throughout the whole recording. $\mathrm{E}$ - Spontaneous short AV block in Cap $2^{g t / 4} . \mathrm{F}-$ Pause of $707 \mathrm{~ms}$ in Cap $2^{\text {gt/gt }}$ : displayed is an initial sinus arrest, followed by a $\mathrm{P}$ wave after $430 \mathrm{~ms}$ that is not conducted to the ventricles as a sign of AV nodal dysfunction. G - Spontaneous ventricular couplets in Cap $2^{\text {gt/gt }}$. H - Idioventricular (junctional) rhythm occurred frequently in Cap $2^{\text {gt/gt }}$, characterized by an initial acceleration in ventricular heart rate, changes in QRS morphology and AV dissociation. P waves are marked by asterisks. Cycle lengths (ms) of RR intervals are given above each QRS complex 
Table I. In vivo electrophysiological investigation

\begin{tabular}{|lcccc|}
\hline Parameter & $\begin{array}{c}\text { WT } \\
n=12\end{array}$ & $\begin{array}{c}\text { Cap } 2^{\text {gt/+ }} \\
n=8\end{array}$ & $\begin{array}{c}\text { Cap } 2^{\text {gt/gt }} \\
n=13\end{array}$ & $\begin{array}{c}\text { One-way ANOVA } \\
p \text {-value }\end{array}$ \\
\hline Cycle length [ms] & $133.6 \pm 8.5$ & $125.0 \pm 14.2$ & $143.8 \pm 14.0^{+}$ & 0.007 \\
\hline Heart rate [bpm] & $450.8 \pm 27.9$ & $485.6 \pm 56.8$ & $421.0 \pm 40.6^{+}$ & 0.006 \\
\hline P [ms] & $15.6 \pm 2.6$ & $14.0 \pm 1.6$ & $17.2 \pm 3.3^{+}$ & 0.042 \\
\hline PQ [ms] & $38.6 \pm 6.5$ & $39.6 \pm 2.1$ & $46.3 \pm 4.1^{*+}$ & 0.001 \\
\hline QRS [ms] & $12.6 \pm 1.4$ & $13.0 \pm 1.1$ & $16.2 \pm 2.6^{*+}$ & $<0.001$ \\
\hline QT [ms] & $45.0 \pm 3.8$ & $44.5 \pm 0.7$ & $57.8 \pm 6.1^{*+}$ & 0.004 \\
\hline QTc [ms] & $45.2 \pm 3.3$ & $47.0 \pm 2.8$ & $55.8 \pm 6.0^{*}$ & 0.015 \\
\hline AH [ms] & $33.9 \pm 6.4$ & $36.2 \pm 4.9$ & $40.6 \pm 10.0$ & NS \\
\hline HV [ms] & $6.5 \pm 3.1$ & $6.5 \pm 2.6$ & $9.7 \pm 2.1^{*}$ & 0.023 \\
\hline WBP [ms] & $92.8 \pm 14.4$ & $90.6 \pm 6.2$ & $95.6 \pm 14.5$ & NS \\
\hline $2: 1$ block [ms] & $52.8 \pm 7.5$ & $56.9 \pm 5.9$ & $55.6 \pm 9.0$ & NS \\
\hline SNRT [ms] & $217.0 \pm 83.5$ & $205.3 \pm 29.3$ & $224.2 \pm 137.5$ & NS \\
\hline AVNRP [ms] & $49.4 \pm 17.6$ & $53.1 \pm 4.6$ & $56.9 \pm 16.7$ & NS \\
\hline ARP [ms] & $29.4 \pm 9.0$ & $35.0 \pm 6.0$ & $32.9 \pm 8.6$ & NS \\
\hline VRP [ms] & $33.9 \pm 12.7$ & $33.1 \pm 12.2$ & $48.8 \pm 20.0$ & NS \\
\hline
\end{tabular}

QTC - heart rate corrected QT time (Mitchell), AH - interval from atrial to His signal, HV - interval from His to ventricular signal, WBP - Wenckebach periodicity; 2: 1 block: 2: 1 AV nodal block, SNRT - sinus node recovery time, AVNRP - AV nodal refractory period, $A R P$ - atrial refractory period, $V R P$ - ventricular refractory period.

${ }^{*} p<0.05$ compared to $W T,+p<0.05$ compared to Cap $2^{g t / t}$.

ent in Cap2 $2^{\text {gt/gt }}$ mice in the 6-lead surface-ECG recordings during the EP study. Moreover, the invasive EP investigations showed that the $P Q$ prolongation resulted from a marked infra-Hisian (HV-time) conduction delay (Table I), i.e. at the level beneath the compact AV node. Functional testing could not detect relevant differences regarding WBP, 2 : 1-block, SNRT, AVNRP, ARP and VRP.

The probability of induction of AF was approximately $16 \%$ in all groups, without significant differences; however, WT mice displayed episodes up to a maximal duration of only 2 min, whereas long-lasting episodes of AF could be seen in Cap$2^{g t / g t}$ (maximum duration $11 \mathrm{~min}$ ).

The inducibility of VTs was significantly increased in Cap $2^{g t /+}$ and Cap $2^{\text {gt/gt }}$ as compared to WT (Figure 2). All ventricular arrhythmia episodes that could be induced in WT mice were short (maximum 4 beats), whereas in mutant mice long-lasting VT episodes up to $1.7 \mathrm{~s}$ could be induced. During the course of the EP investigations one Cap $2^{g t / t}$ mouse died from a $3^{\text {rd }}$ degree AV block that developed after a ventricular stimulation maneuver.

The inducibility of VTs in the mutant mice showed a significant negative correlation with VRP (Spearman $r=-0.72 ; p<0.01$ ). No VTs could be induced in mice with a VRP $>55 \mathrm{~ms}$ (Figure $2 \mathrm{D}$ ).

\section{Inactivation of CAP2 leads to slowing of conduction}

We performed ex vivo epicardial mapping of the whole heart preparations to analyze local conduction velocities (Figure 3). Compared to WT, significantly slower epicardial left ventricular conduction velocities in longitudinal fiber orientation could be registered in mutant mice (Cap $2^{g t / t}: 47.0$

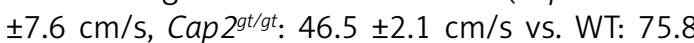
$\pm 10.1 \mathrm{~cm} / \mathrm{s} ; p<0.01)$. Analogously, conduction velocity in transversal fiber orientation was impaired in both mutant groups. Right ventricular epicardial impulse propagation was reduced in Cap $2^{\text {gt/ }}$ and Cap $2^{\text {gt/gt }}$ to a comparable extent in longitudinal and in transversal fiber orientation (Cap $2^{\text {gt/t}}: 49.2$ $\pm 3.9 \mathrm{~cm} / \mathrm{s}$, Cap2 $^{\text {gt/gt }}: 47.0 \pm 14.5 \mathrm{~cm} / \mathrm{s}$ vs. WT: 75.3 $\pm 3.4 \mathrm{~cm} / \mathrm{s} ; p<0.01)$.

\section{Alterations in cardiac connexins accompany arrhythmia susceptibility}

To further evaluate the mechanism of arrhythmogenesis associated with CAP2 deletion, we performed an analysis of cardiac connexins. We determined mRNA levels of $\mathrm{Cx} 40$ in the AV junction as CX40 is expressed in the AV node and the specific conduction system. In Cap $2^{g t / 4}$ we detected up-regulation of $\mathrm{Cx} 40$, whereas Cap $2^{\text {gt/gt }}$ showed opposite results: Here, Cx40 mRNA levels were significantly reduced compared to WT (Figure 4). We additionally analyzed $\mathrm{C} \times 40$ protein expression at the atrial level. The results pointed in the same direction as the results from the mRNA expression analysis in the AV junction, though not reaching the level of significance, indicating that predominant changes originate from the specific cardiac conduction system and not from atrial tissue. 
A

WT
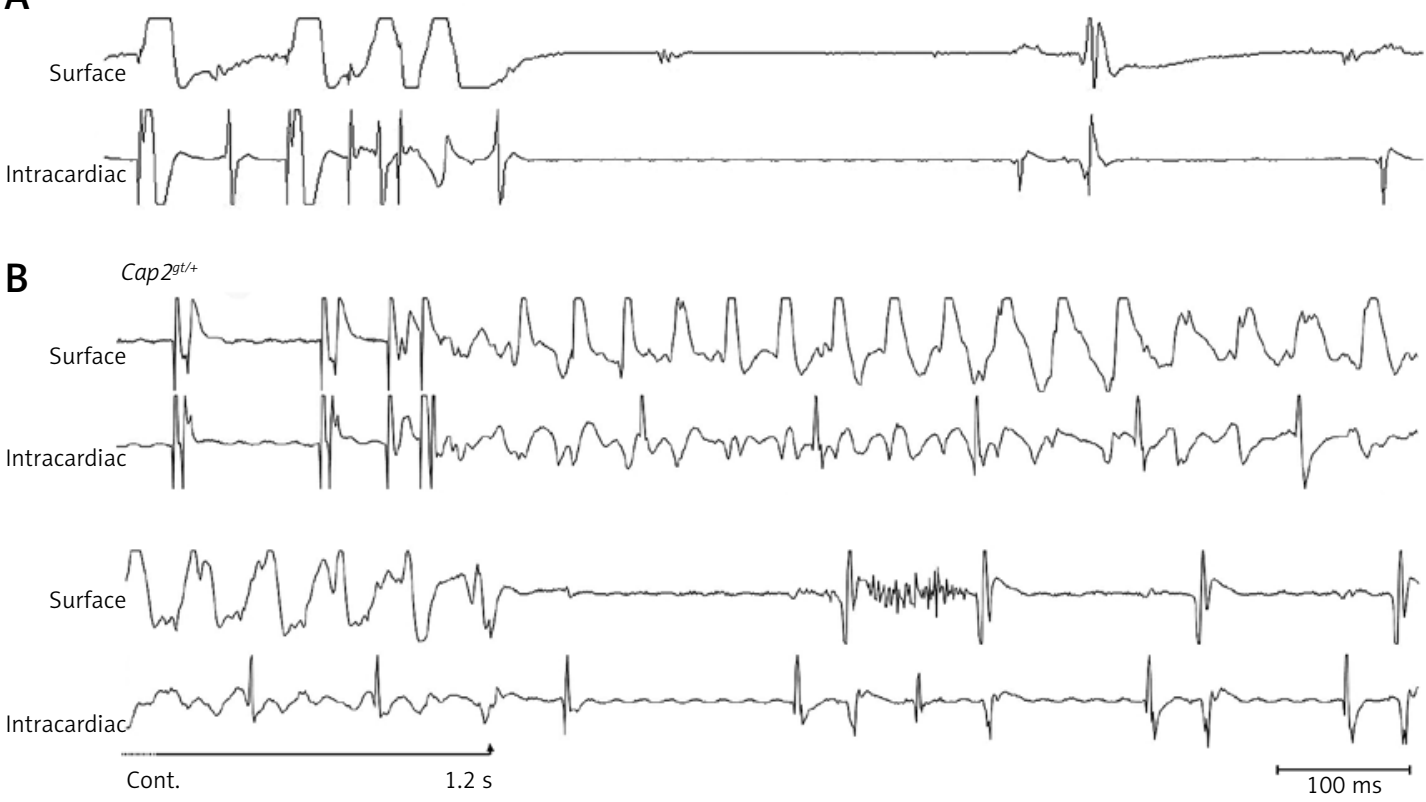

C
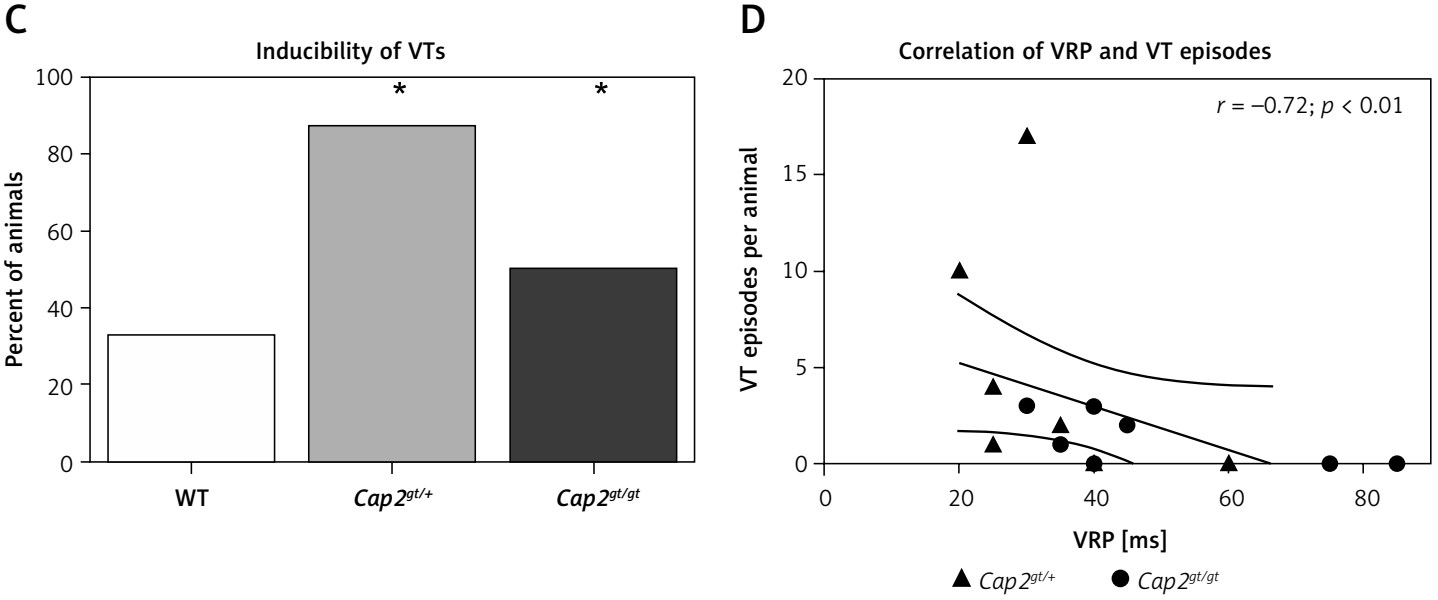

Figure 2. Induction of ventricular tachycardias. Representative ECG recordings of WT (A) and Cap $2^{\text {gt/ }}$ mice (B). Ventricular extrastimulus pacing close to the refractory period did not lead to the induction of an arrhythmia in WT (S1S2: $50 \mathrm{~ms}$, S2S3: $35 \mathrm{~ms}$ ). In Cap 2gt/ the pacing maneuver (S1S2: $50 \mathrm{~ms}, \mathrm{~S} 2 \mathrm{S3}: 25 \mathrm{~ms}$ ) led to the induction of a ventricular tachycardia (VT) lasting over $1200 \mathrm{~ms}$. C - The inducibility of VTs was significantly elevated in the mutant mice compared to WT. D - The number of VT episodes per animal was significantly negatively correlated with the ventricular refractory period (VRP). Mutant mice with longer refractory periods exhibited fewer VT episodes

$n=8-9$ per group. ${ }^{*} p<0.05$ vs. WT.

Analysis of ventricular Cx43 revealed significant alterations among the investigated an-

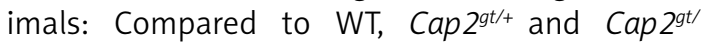
gt presented significantly up-regulated levels of Cx43 mRNA, predominantly in the left ventricle (Figure 5). At the protein level, this did not lead to an increase in elevated levels of $\mathrm{Cx} 43$ protein, indicating posttranscriptional regulation defects. No differences in the status of phosphorylated in comparison to non-phosphorylated $\mathrm{Cx} 43$ was found in the western blot analysis. The cellular localization of Cx43 was markedly altered, as shown by immunostaining: whereas in Cap $2^{\text {gt/ }}$ the distribution pattern was still similar to WT with predominant localization at the intercalated discs and few signs of lateralization, in Cap $2^{\text {gt/gt }}$ the Cx43 signal showed a significant quantitative increase in dispersed punctuate gap junctions distributed along lateral boundaries of the myocytes (maldistribution of Cx43 in Cap $2^{g t / 4}$ : $10.9 \pm 2.9 \%$, Cap 2 $^{\text {gt/gt }}: 23.0 \pm 4.7 \%$ vs. WT: $2.9 \pm 0.8 \%$; $p<0.0001$ ) (Figure 5).

\section{Structural remodeling is present in hearts of Cap2 ${ }^{\text {gt/gt }}$ mice}

F-actin staining by phalloidin revealed that in many distinct parts of the heart F-actin assem- 
A

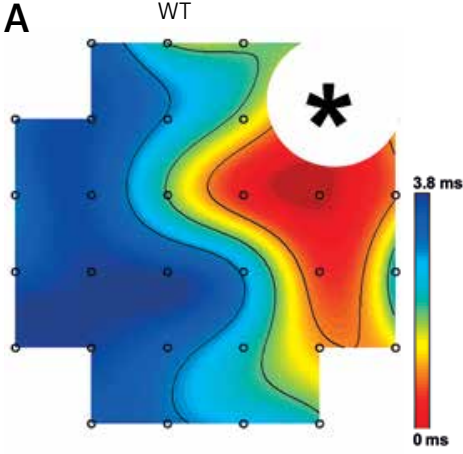

Cap $2^{g t /+}$

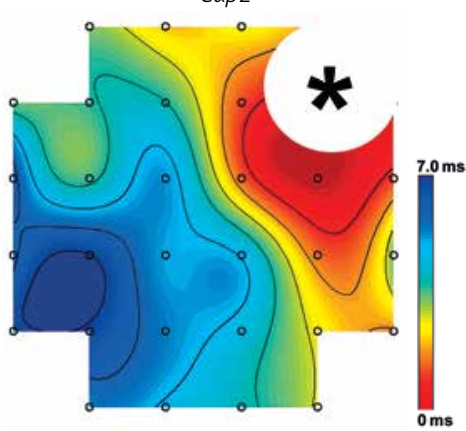

Cap $2^{g t / g t}$

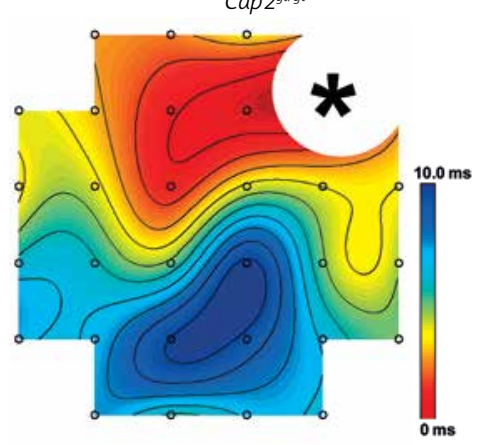

B

Conduction velocities after epicardial stimulation
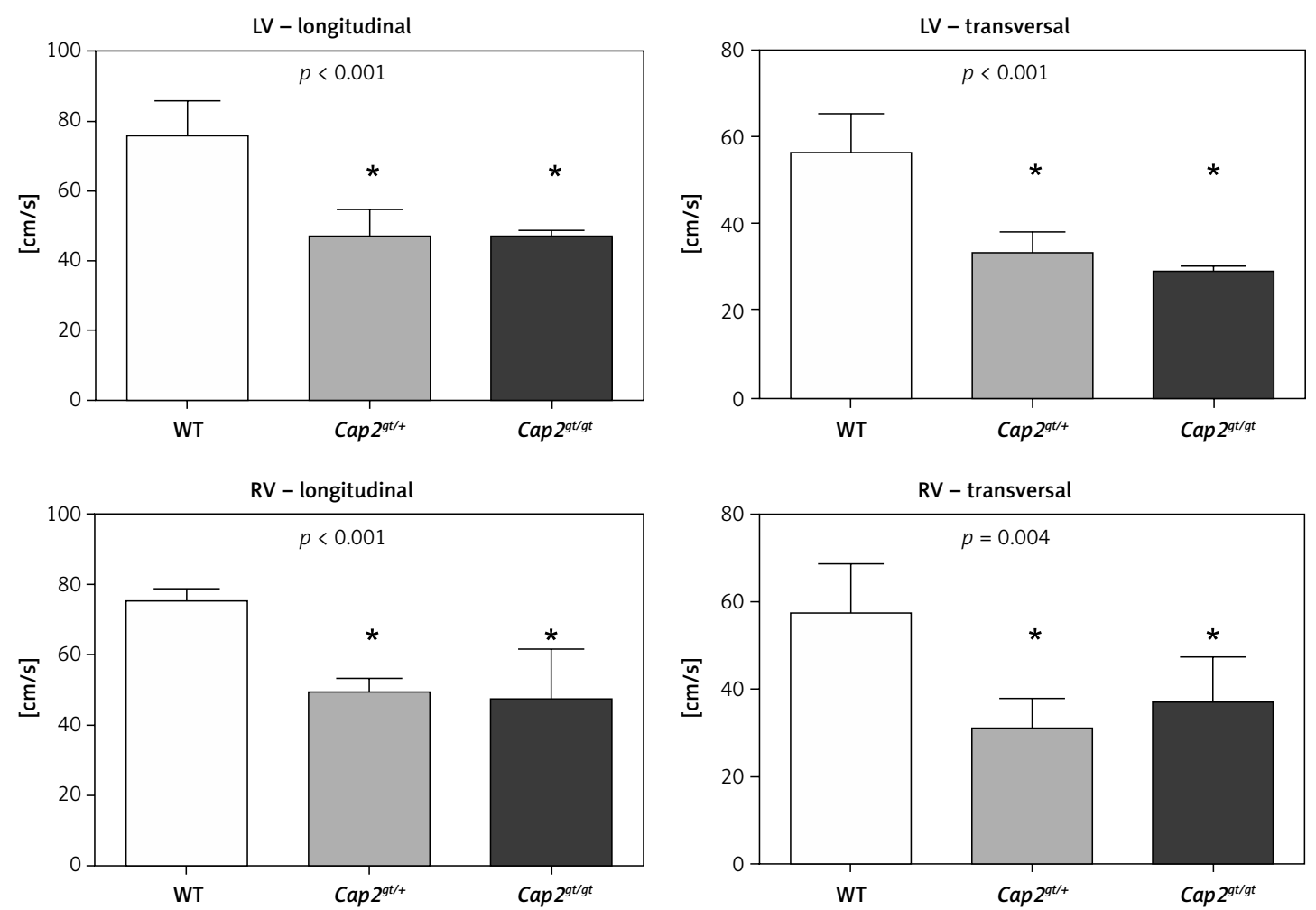

Figure 3. Epicardial mapping. A - Representative epicardial activation maps after applied pacing from the left ventricle. Isochrones are illustrated by black lines, the distance between each line representing 1 ms delay. Accumulation of isochrones as shown in Cap $2^{g t /+}$ and Cap $2^{g t / g t}$ indicates local slowing of conduction. The area of stimulus application is marked with an asterisk. B - Both mutant groups revealed a significant slowing of conduction in longitudinal and transversal myocardial fiber orientation in the left (LV) and right ventricle (RV)

${ }^{*} p<0.05$ compared to WT. WT: $n=4$, Cap $2^{g t / t}: n=4$, and Cap $2^{g t / g t}: n=3$.

bly in the sarcomere was disturbed (Figure 5). In accordance with local slowing of conduction in epicardial mapping, we detected a significant increase in myocardial fibrosis by Sirius red staining in hearts of Cap $2^{\text {gt/gt }}$ mice (Figure 6). This was particularly prominent in the left ventricle, whereas the right ventricle appeared unchanged compared to WT. In heterozygous Cap2 hearts no relevant alterations in myocardial fibrosis quantity were measurable.

Analogous to the study by Peche et al. [6], we found a decrease of $8.6 \%$ in body weight of the Cap $2^{\text {gt/gt }}$ mice compared to WT, resulting in a sig- nificant increase in the heart weight / body weight ratio (Table II).

\section{Discussion}

The previously described phenotype of a biventricular cardiomyopathy [6] that is seen in mice with a Cap2 gene ablation is associated with marked changes in cardiac electrophysiological parameters. We detected significant bradycardia, AV conduction delays and ventricular arrhythmias.

Local slowing of conduction represents an established pathomechanism for the induction and perpetuation of malignant arrhythmias [11]. Car- 
A

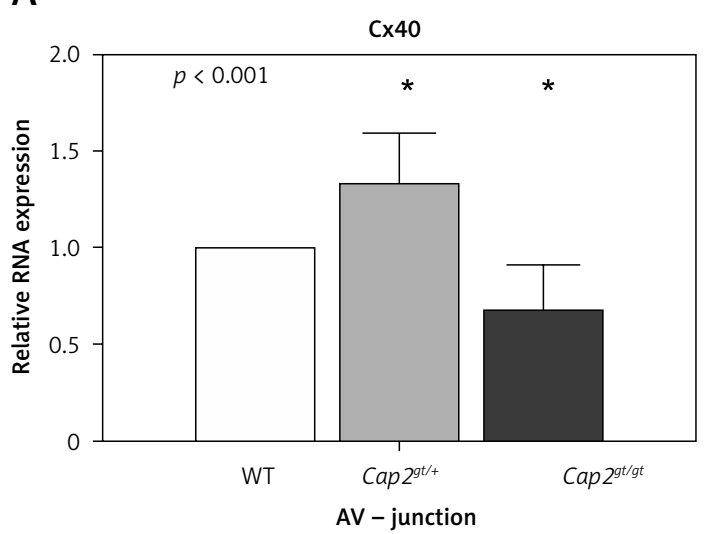

B

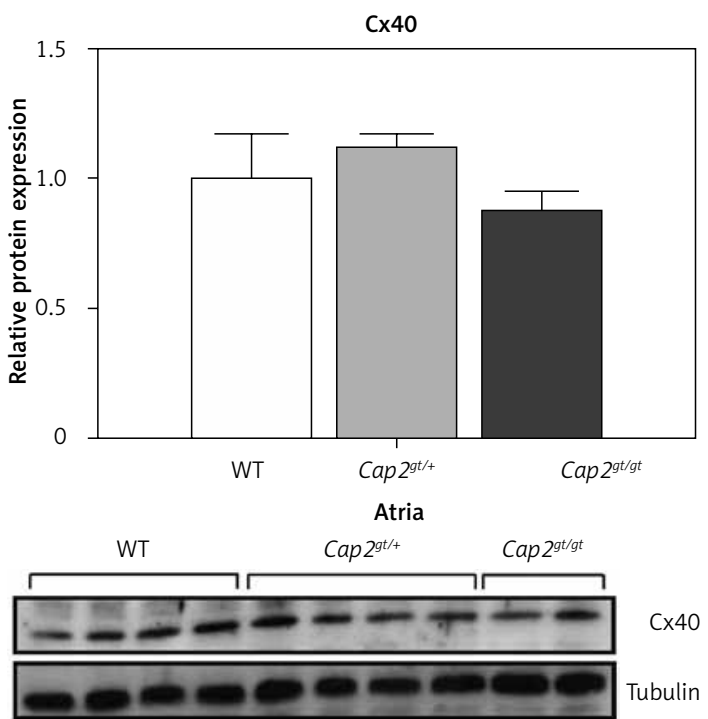

Figure 4. Quantification of connexin40. A - In the AV junction Cx40 mRNA was up-regulated only in Cap $2^{g t /+}$ but significantly down-regulated in Cap $2^{\text {gt/gt }}$. B - The results from the protein expression of Cx40 in the atria showed no significant changes between the groups, indicating that $\mathrm{Cx} 40$ changes result from alterations in the specific cardiac conduction system and not from atrial tissue

$L V$ - left ventricle, $R V$ - right ventricle, $n=5-6$ per group. ${ }^{*} p<0.05$ compared to WT.

diac conduction velocity is markedly influenced by cell-to-cell coupling and tissue structure [12].

Regarding cell-to-cell coupling, we detected marked alterations in $\mathrm{Cx} 43$ distribution. Normal function of the actin cytoskeleton has been shown to be essential for Cx43 trafficking, and inhibition of actin polymerization is sufficient to reduce levels of $\mathrm{Cx} 43$ gap junctions at intercalated discs [13]. Moreover, a sole destabilization of the cardiac cytoskeleton has been shown to directly interfere with cardiac connexins and resulted in an abnormal $\mathrm{Cx} 43$ redistribution and loss of gap junction plaque [14]. The relative increase of lateral Cx43 accumulation did not lead to an increase in transversal conduction velocity in our model. This is consistent with the results of other studies in dilated cardiomyopathy that showed that although being phosphorylated, the lateralized Cx43 is non- or hypofunctional and conduction velocity is uniformly reduced in the longitudinal and transversal direction of impulse propagation $[15,16]$. A further reason for a decrease in transversal conduction velocity might be the progressive intercellular fibrosis found in our mutant mice which is, relative to the same area, proportionately more pronounced in transversal fiber orientation as a result of the shorter cell dimensions.

Homozygous Cap 2 mice exhibited marked tissue structure alterations as indicated by increases in relative heart size as well as increase in myocardial fibrosis compared to WT. Myocardial fibrosis contributes to altered tissue architecture, resulting in reduced myocyte-myocyte coupling and formation of fibroblast-myocyte coupling [17]. In consequence, this provokes conduction delays via a zigzag course of electrical impulse propagation, which has been shown to predispose to re-entry arrhythmias [18]. These anatomical disruptions have a further impact on slowing of conduction and enhance the effect of connexin distributive abnormalities.

What is more, sole alterations in $\mathrm{C} \times 43$ function have been shown to impact on arrhythmogenesis [19, 20]. Interestingly, despite not exhibiting an increase in fibrosis compared to WT and presenting with only $10.9 \%$ of abnormal distribution of Cx43, Cap $2^{\text {gt/t }}$ mice showed marked reductions in conduction velocities. This places emphasis on the proper distribution and functioning of cardiac connexins.

The inducibility of VTs is closely linked to the ventricular refractory period (VRP), since longer VRPs to a certain extent result in an antiarrhythmic effect [21]. Mice with the homozygous Cap2 inactivation show a great interindividual range of the VRP. Mice with longer VRPs (the cut-off value in this study was $55 \mathrm{~ms}$ ) were not susceptible to VTs. Peche et al. [6] reported that Cap $2^{\text {gt/gt }}$ mice exhibit a reduced life span and $\sim 63 \%$ of the male animals died suddenly and spontaneously before the age of 70 days. Interestingly, the remaining mice survived up to one year. Even though Cap2 was inactivated in all Cap $2^{\text {gt/gt }}$ mice, and all these animals exhibited a dilatative cardiomyopathy phenotype, this points towards additional electrophysiological alterations involved in the suscepti- 
A
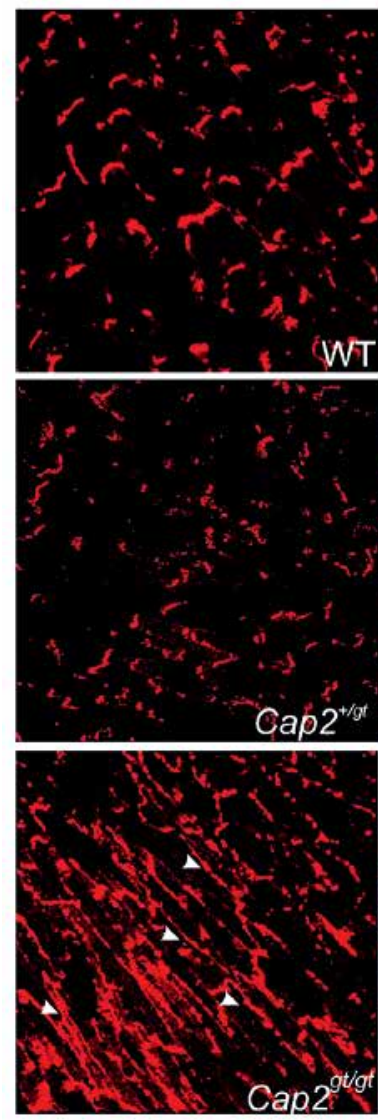

B
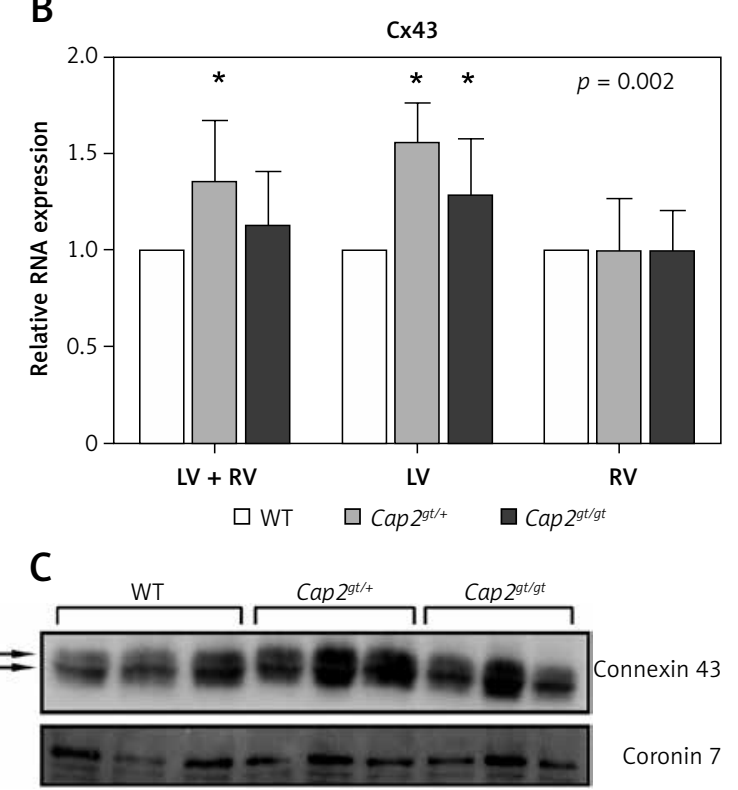

Connexin 43

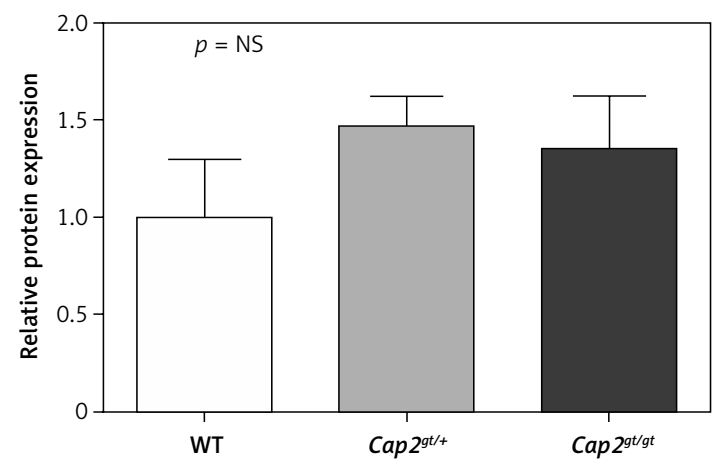

Merge
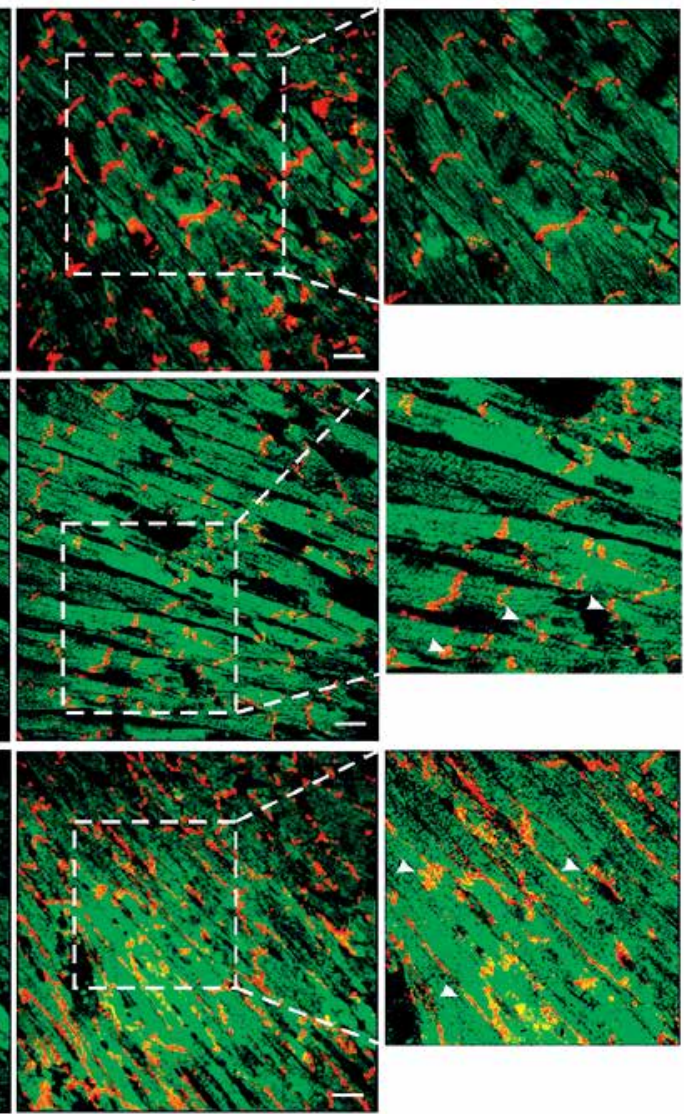

D

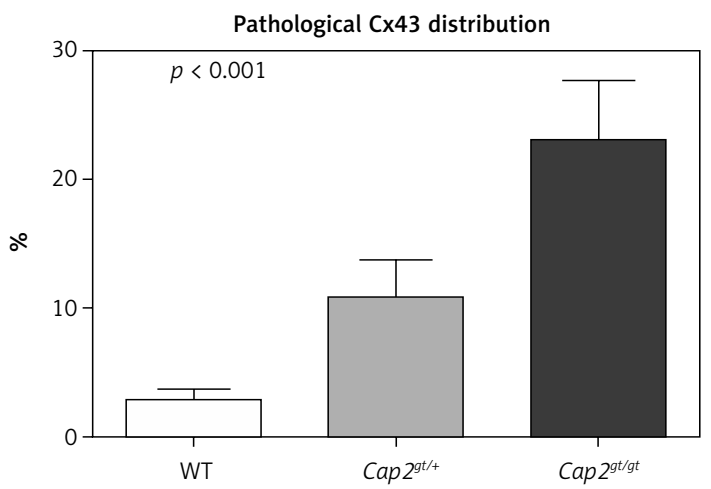

Figure 5. Distribution of connexin43. A - Immunostaining of ventricular sections showed localization of connexin43 (Cx43) with intense staining plaques at the intercalated discs in WT mice. In Cap $2^{\text {gt/ }}$ the Cx43 signal was still present at the intercalated discs but showed the first signs of lateralization. Marked maldistribution and lateralization of the punctuate Cx43 signal were present in Cap $2^{\text {gt/gt }}$ (arrowheads). Co-staining with phalloidin revealed that F-actin assembly in the cardiomyocytes was severely disturbed. Green = phalloidin, red $=$ Cx43, bar $=20 \mu \mathrm{m}$. B - Semiquantitative real-time PCR analysis revealed up-regulation of $\mathrm{Cx} 43$ in the mutant mice, which was predominantly due to alterations in the left ventricle. $\mathbf{C}$ - Western blots of $\mathrm{C} \times 43$ showed no quantitative differences in ventricular protein concentrations between the groups. Arrows indicate the phosphorylated $\mathrm{C} \times 43$ bands. There was no evidence of relevant non-phosphorylated Cx43 accumulation in any group. D - A marked increase in lateralized Cx43 distribution was present in Cap $2^{\text {gt/gt }}$ and, with weaker expression, in Cap $2^{g t /+}$ in comparison to WT

$L V$ - left ventricle, $R V$ - right ventricle, ${ }^{*} p<0.05$ compared to WT. $n=5$ per group. 
A

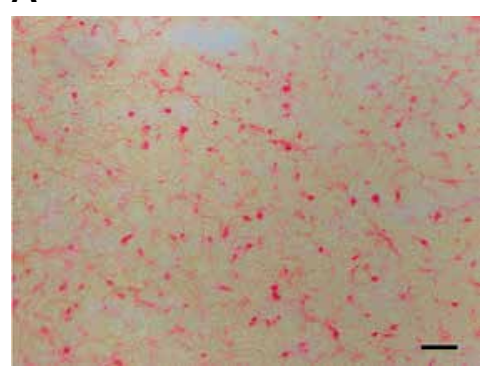

$\operatorname{Cap} 2^{g t /+}$

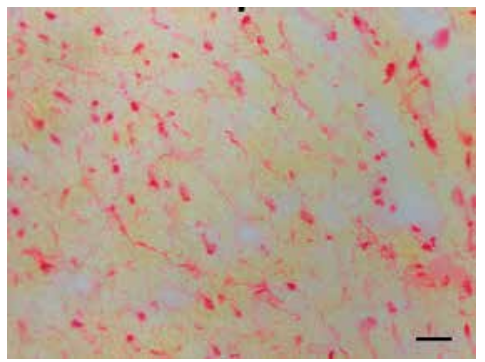

$\operatorname{Cap} 2^{g t / g t}$

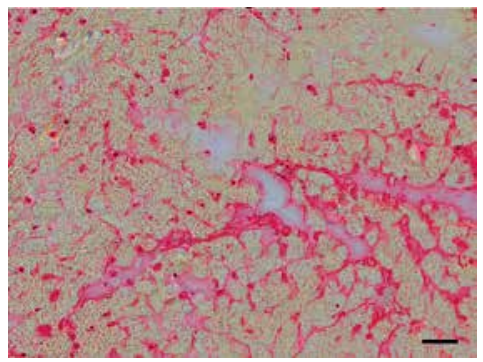

B

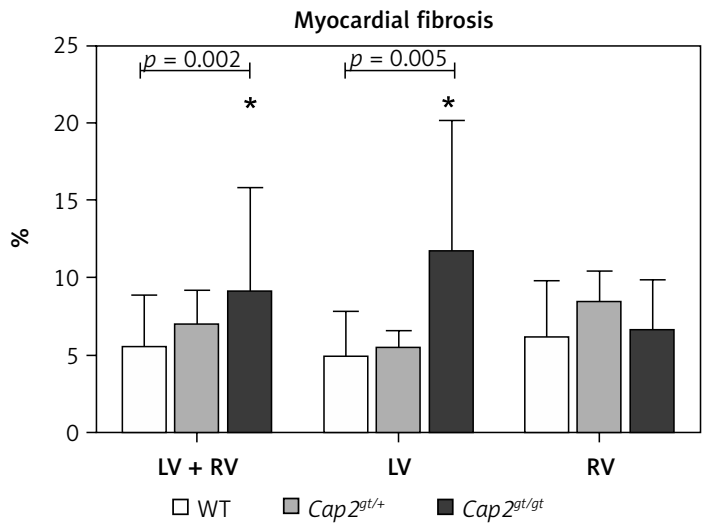

bility to malignant arrhythmias. The longer VRPs in individual animals are one of such potential mechanisms.

The alterations in Cx40 expression in homozygous Cap $2^{\text {gt/gt }}$ are consistent with AV conduction delays and spontaneous AV blocks, since Cx40 is a major connexin component of the specific cardiac conduction system [22]. However, Cap $2^{g t /+}$ heterozygotes exhibit an increase in Cx40 expression. The heterozygous gene inactivation does not lead to increased fibrosis or major myocardial dilatation, and analogously also does not seem to have a negative impact on Cx40 expression and conduction properties of the specific cardiac conduction system. Therefore, the Cx40 up-regulation found in the heterozygous animals is likely to represent an effective compensatory mechanism.

Mutations in the actin cytoskeleton are frequently found in congenital myopathies, including dilated or hypertrophic cardiomyopathies and left ventricular non-compaction. Histological analysis in patients suffering from actinopathies reveal an increase in fibrosis [23], which we also detected in

\begin{abstract}
Figure 6. Myocardial fibrosis. A - Sirius red staining of left ventricular heart sections. An increase in red stained intercellular connective tissue could be detected in Cap $2^{g t / g t}$. Bar $=20 \mu \mathrm{m}$. B - Pixel analysis revealed a significant increase in ventricular fibrosis only in Cap $2^{g t / g t}$

$L V$ - left ventricle, $R V$ - right ventricle, ${ }^{*} p<0.05$ compared to WT; WT: $n=8$, Cap2 $2^{g t / t}: n=4$, and Cap $2^{g t / g t}: n=4$.
\end{abstract}

the mutant mice. It has been shown that myocardial fibrosis represents a prognostic risk parameter with increased mortality in patients with dilated cardiomyopathies [24]. Since inactivation of CAP2 leads to a disruption of the G-actin/F-actin equilibrium and therefore severely affects a proper cardiac cytoskeleton, Cap2 mutations might represent a so far unrecognized cause of cardiomyopathies.

The involvement in human disease is further supported by its gene locus at chromosome $6 p 22.3$. The interstitial $6 p 22$ deletion syndrome and copy number variations at $6 \mathrm{p} 22$ lead to multiple cardiac abnormalities [25] and sudden infant death [26]. Though until now no cardiomyopathy has been associated with CAP2, a possible relationship should be addressed in further studies of human cardiomyopathies. Consistent with the aforementioned syndromes that both represent haploinsufficiency defects, we found a considerable incidence of malignant ventricular arrhythmias even in the heterozygous Cap $2^{g t /+}$ animals. Subsequent studies should therefore focus on

Table II. Physiological parameters

\begin{tabular}{|lcccc|}
\hline Parameter & $\begin{array}{c}\text { WT } \\
n=12\end{array}$ & $\begin{array}{c}\text { Cap2 } 2^{\text {gt/ }} \\
n=8\end{array}$ & $\begin{array}{c}\text { Cap2 } 2^{g t / g t} \\
n=13\end{array}$ & $\begin{array}{c}\text { One-way ANOVA } \\
p \text {-value }\end{array}$ \\
\hline Body weight $[\mathrm{g}]$ & $27.0 \pm 2.2$ & $26.7 \pm 2.9$ & $24.1 \pm 2.3^{*}$ & 0.016 \\
\hline Heart weight $[\mathrm{g}]$ & $0.1644 \pm 0.024$ & $0.1775 \pm 0.0269$ & $0.1808 \pm 0.0351$ & NS \\
\hline HW/BW & $0.00625 \pm 0.0006$ & $0.0066 \pm 0.0007$ & $0.00724 \pm 0.0012^{*}$ & 0.028 \\
\hline
\end{tabular}

$H W$ - heart weight, BW-body weight. ${ }^{*} p<0.05$ compared to WT. 
human patients with undetermined conduction disturbances, ventricular arrhythmias or cardiomyopathies, to reveal Cap 2 mutations as a possible causative pathomechanism in human disease.

In conclusion, the inactivation of Cap2 results in a cardiomyopathy with relevant electrophysiological alterations, predominantly spontaneous and inducible malignant ventricular arrhythmias. These are accompanied by impaired sinus node function and conduction disturbances. Alterations in the expression of connexins and myocardial fibrosis contribute to the enhanced susceptibility to arrhythmias. Evaluation of Cap2 mutations in human patients suffering from heart failure, dilative cardiomyopathies, as well as primary ventricular tachycardias or sudden arrhythmogenic death, might reveal a new pathomechanism for arrhythmogenesis.

\section{Acknowledgments}

This work was supported by a research grant from the German Cardiac Society awarded to FS (DGK Stipendium 2012) and a research grant from the University of Cologne 'Köln Fortune' awarded to VSP.

\section{Conflict of interest}

The authors declare no conflict of interest.

\section{References}

1. Pollard TD. The cytoskeleton, cellular motility and the reductionist agenda. Nature 2003; 422: 741-5.

2. Freeman NL, Chen Z, Horenstein J, Weber A, Field J. An actin monomer binding activity localizes to the carboxyl-terminal half of the Saccharomyces cerevisiae cyclase-associated protein. J Biol Chem 1995; 270: 5680-5.

3. Noegel AA, Rivero F, Albrecht R, et al. Assessing the role of the ASP56/CAP homologue of Dictyostelium discoideum and the requirements for subcellular localization. J Cell Sci 1999; 112: 3195-203.

4. Bertling E, Hotulainen P, Mattila PK, Matilainen T, Salminen $M$, Lappalainen P. Cyclase-associated protein 1 (CAP1) promotes cofilin-induced actin dynamics in mammalian nonmuscle cells. Mol Biol Cell 2004; 15 2324-34.

5. Peche V, Shekar S, Leichter M, et al. CAP2, cyclase-associated protein 2 , is a dual compartment protein. Cell Mol Life Sci 2007; 64: 2702-15.

6. Peche VS, Holak TA, Burgute BD, et al. Ablation of cyclase-associated protein 2 (CAP2) leads to cardiomyopathy. Cell Mol Life Sci 2013; 70: 527-43.

7. Morimoto S. Sarcomeric proteins and inherited cardiomyopathies. Cardiovasc Res 2008; 77: 659-66.

8. Finsterer J, Stollberger C, Keller H. Arrhythmia-related workup in hereditary myopathies. J Electrocardiol 2012; 45: 376-84.

9. Schrickel JW, Brixius K, Herr C, et al. Enhanced heterogeneity of myocardial conduction and severe cardiac electrical instability in annexin A7-deficient mice. Cardiovasc Res 2007; 76: 257-68.
10. Rybakin V, Stumpf M, Schulze A, Majoul IV, Noegel AA, Hasse A. Coronin 7, the mammalian POD-1 homologue, localizes to the Golgi apparatus. FEBS Lett 2004; 573: 161-7.

11. Gutstein DE, Morley GE, Tamaddon H, et al. Conduction slowing and sudden arrhythmic death in mice with cardiac-restricted inactivation of connexin43. Circ Res 2001; 88: 333-9.

12. Kleber AG, Rudy Y. Basic mechanisms of cardiac impulse propagation and associated arrhythmias. Physiol Rev 2004; 84: 431-88.

13. Smyth JW, Vogan JM, Buch PJ, et al. Actin cytoskeleton rest stops regulate anterograde traffic of connexin 43 vesicles to the plasma membrane. Circ Res 2012; 110 : 978-89.

14. Oxford EM, Musa H, Maass K, Coombs W, Taffet SM, Delmar M. Connexin43 remodeling caused by inhibition of plakophilin-2 expression in cardiac cells. Circ Res 2007; 101: 703-11.

15. Akar FG, Nass RD, Hahn S, et al. Dynamic changes in conduction velocity and gap junction properties during development of pacing-induced heart failure. Am J Physiol Heart Circ Physiol 2007; 293: H1223-30.

16. Akar FG, Spragg DD, Tunin RS, Kass DA, Tomaselli GF. Mechanisms underlying conduction slowing and arrhythmogenesis in nonischemic dilated cardiomyopathy. Circ Res 2004; 95: 717-25.

17. Camelliti P, Green CR, LeGrice I, Kohl P. Fibroblast network in rabbit sinoatrial node: structural and functional identification of homogeneous and heterogeneous cell coupling. Circ Res 2004; 94: 828-35.

18. de Bakker JM, van Capelle FJ, Janse MJ, et al. Slow conduction in the infarcted human heart. 'Zigzag' course of activation. Circulation 1993; 88: 915-26.

19. Roell W, Lewalter T, Sasse P, et al. Engraftment of connexin 43-expressing cells prevents post-infarct arrhythmia. Nature 2007; 450: 819-24.

20. Liu Y, Li H, Xia W, Yu S, Huang C, Huang H. Electrophysiological effect of rotigaptide in rabbits with heart failure. Arch Med Sci 2014; 10: 374-80.

21. Waldeyer C, Fabritz L, Fortmueller L, et al. Regional, age-dependent, and genotype-dependent differences in ventricular action potential duration and activation time in 410 Langendorff-perfused mouse hearts. Basic Res Cardiol 2009; 104: 523-33.

22. Kirchhoff S, Kim JS, Hagendorff A, et al. Abnormal cardiac conduction and morphogenesis in connexin 40 and connexin43 double-deficient mice. Circ Res 2000; 87: 399-405.

23. Wallgren-Pettersson C, Rapola J, Donner M. Pathology of congenital nemaline myopathy. A follow-up study. J Neurol Sci 1988; 83: 243-57.

24. Gulati A, Jabbour A, Ismail TF, et al. Association of fibrosis with mortality and sudden cardiac death in patients with nonischemic dilated cardiomyopathy. JAMA 2013, 309: 896-908.

25. Davies AF, Mirza G, Sekhon G, et al. Delineation of two distinct $6 p$ deletion syndromes. Hum Genet 1999; 104 64-72.

26. Toruner GA, Kurvathi R, Sugalski R, et al. Copy number variations in three children with sudden infant death. Clin Genet 2009; 76: 63-8. 